\title{
Refined Phase Imaging by Electron Diffractive Imaging
}

\author{
Jun Yamasaki ${ }^{1}$, Yuki Shimaoka ${ }^{2}$ and Hirokazu Sasaki ${ }^{3}$ \\ 1. Research Center for Ultra-High Voltage Electron Microscopy, Osaka University, Ibaraki, Osaka, \\ Japan. \\ 2. Graduate School of Engineering, Osaka University, Ibaraki, Osaka, Japan. \\ 3. Furukawa Electric Co., Ltd, Yokohama, Kanagawa, Japan.
}

Phase imaging of transmission electron beams is indispensable in visualizing electromagnetic fields in and around nanometer-sized semiconducting or magnetic devices as well as detecting sample thickness and composition based on its mean inner potential. Although the most established method for the phase imaging is presently off-axis electron holography, another choice could be electron diffractive imaging (EDI). In the method, a complex wave field is reconstructed from a diffraction pattern through numerical iterations under some constraints. So far we have succeeded in reconstructions of atomic structures of crystals [1,2], thickness maps of wedge-shaped Si [3] and a p-n junction [3,4].

Figure 1 shows a simple example, in which the phase image of an edge of an antigorite crystal supported on a carbon film is reconstructed based on the data set of the TEM image with a selector aperture (Fig. 1(a)) and the corresponding diffraction pattern (Fig. 1(b)). In Fig. 1(c), unnatural fringe and modulation of phase have appeared in the area of the uniform carbon film. The probable cause of the problem is that the measured diffraction pattern is modified from the ideal Fourier transform of the sample structure. The modification should be mainly caused by 1) blurring of the diffraction pattern by partial spatial coherence of the electron beam and MTF of the detector, and 2) aberrations, mainly, of the condenser and intermediate lenses, all of which are known to be important factors also in electron holography.

For quantitative phase reconstructions, we must measure these factors precisely and then eliminate their influences from the reconstructed phase images. In an electron microscope unequipped with an electron biprism, measurements of them may be not impossible [5,6] but generally time-consuming tasks. In the present study, we developed a more straightforward method to measure them based on fitting calculations. As shown in Fig. 2, the Airy diffraction pattern using a selector aperture inserted in a vacuum area (Fig. 2(a)) is reproduced very well by the calculated pattern (Fig. 2(b)) using some aberration coefficients and a point spread function (PSF) with Gaussian distribution, which are listed in Fig. 2(c). The influence of the PSF can be eliminated by a deconvolution process of the diffraction pattern. On the other hand, the additional phase shift induced by the lens aberrations can be subtracted simply from the reconstructed phase image. After these processes, we finally obtained the phase image in Fig. 1(d), which shows a flat phase distribution reflecting the uniform carbon film. The phase fluctuation in the carbon film area is about 0.1-0.2 rad, which is comparable to electron holography.

In addition to the unnecessity of a biprism, the notable advantage over off-axis electron holography is that the present phase imaging method based on EDI does not need a vacuum area adjoining to the field of view of interest. Therefore, phase images of complicated sample structures far from vacuum areas can be easily observed as shown in Fig. 3. This advantage exhibits a future possibility that the phase imaging based on EDI will become an alternative to electron holography in some cases, for example, in observations of semiconductor dopant profiles far from the sample edge and observations of various 
dielectric and magnetic materials without disturbed by stray electromagnetic fields extending around samples [7].

\section{References:}

[1] S. Morishita, J. Yamasaki, et al, Appl. Phys. Lett. 93 (2008) 183103.

[2] S. Morishita, J. Yamasaki, et al, AMTC Lett. 2 (2010) 116.

[3] J. Yamasaki, et al, Appl. Phys. Lett. 101 (2012) 234105.

[4] J. Yamasaki, et al, AMTC Lett. 3 (2012) 164.

[5] S. Morishita, J. Yamasaki, et al, J. Electron Microsc. 60 (2011) 101.

[6] S. Morishita, J. Yamasaki, et al, Ultramicrosc. 129 (2013) 10.

[7] The present study was partly supported by JSPS KAKENHI (Grant No. 26286049, 26105009 and 21760026), MEXT KAKENHI (Grant No. 26600042), The Public Foundation of Chubu Science and Technology Center, and Toyoaki Scholarship Foundation.
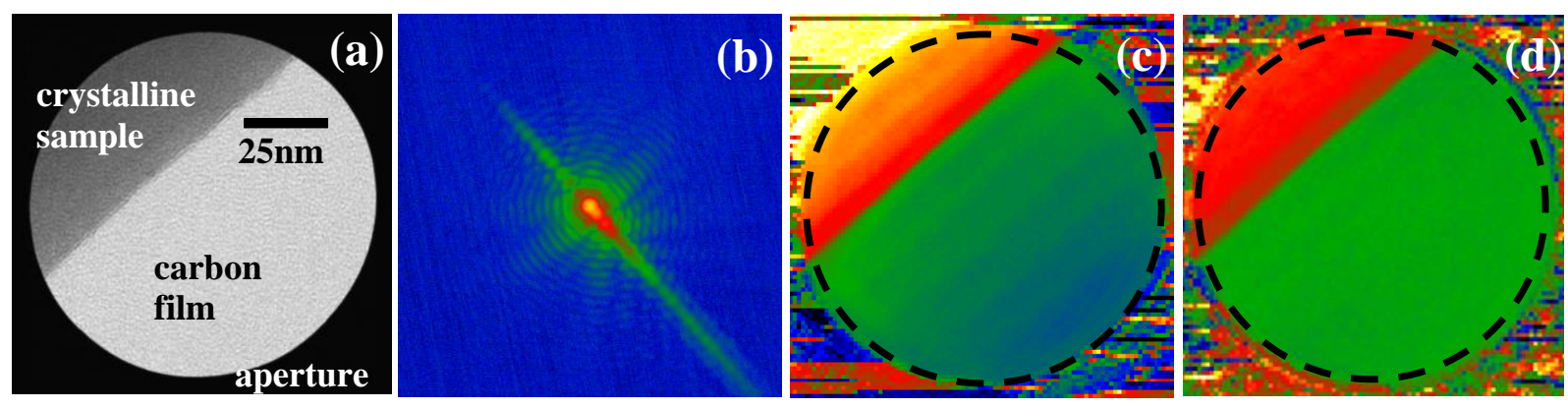

Figure 1. Phase reconstruction of the sample edge on a carbon film. (a) Bright-field TEM image with a selector aperture, (b) the corresponding selected-area diffraction pattern. (c) and (d) show the reconstructed phase image before and after the correction processes, respectively.

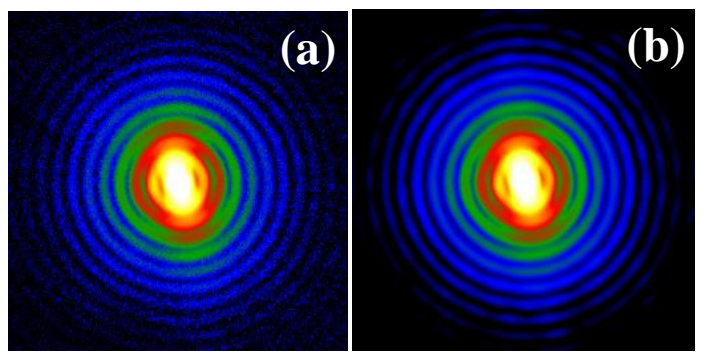

(c)

\begin{tabular}{|l|l|l|}
\hline & Magnitude & Angle \\
\hline Defocus $\left(\mathrm{C}_{1}\right)$ & $-58.0 \mathrm{~m}^{-1}$ & - \\
\hline Two-fold astigmatism $\left(\mathrm{A}_{1}\right)$ & $24.4 \mathrm{~mm}^{-1}$ & $-25.3 \mathrm{deg}$ \\
\hline Three-fold astigmatism $\left(\mathrm{A}_{2}\right)$ & $-630 \mathrm{~m}^{-1}$ & $-12.0 \mathrm{deg}$ \\
\hline Coma aberration $\left(\mathrm{B}_{2}\right)$ & $-12.9 \mathrm{~m}^{-1}$ & $47.8 \mathrm{deg}$ \\
\hline Standard deviation of PSF & $6.29 \mu \mathrm{m}^{-1}$ & - \\
\hline
\end{tabular}

Figure 2. Determination of blurring and lens aberrations included in the Airy pattern. (a) Experimental pattern and (b) fitting calculation using the parameters listed in (c).

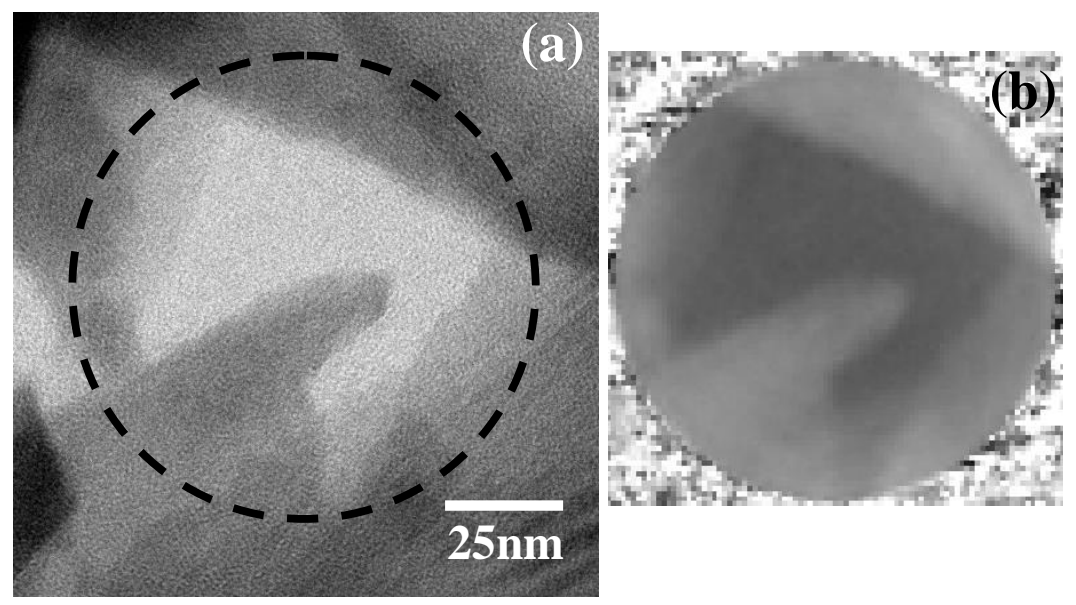

Figure 3. Phase imaging of a complicated sample structure far from vacuum areas. (a) TEM image, (b) the reconstructed phase image. 